\title{
Improvement of the experimental content in Laser Principle and its Application
}

Chun-hui Niu, Xiao-ping Lang, Yong Lv, Hao Meng

Chun-hui Niu, Xiao-ping Lang, Yong Lv, Hao Meng, "Improvement of the experimental content in Laser Principle and its Application," Proc. SPIE 10452, 14th Conference on Education and Training in Optics and Photonics: ETOP 2017, 1045250 (16 August 2017); doi: 10.1117/12.2269589

SPIE Event: 14th Conference on Education and Training in Optics and Photonics, ETOP 2017, 2017, Hangzhou, China 


\title{
Improvement of the experimental content
}

\section{in Laser Principle and Its Application}

\author{
NIU Chun-hui** , LANG Xiao-ping, LV Yong , MENG Hao \\ (School of Instrument Science and Opto Electronics Engineering, Beijing Information \& Technology \\ University, Beijing 100192, China)
}

\begin{abstract}
Experimental teaching content of Laser Principle and Its Application is proposed to improve from experimental teaching devices and experimental guide book. At first, a experimental system of laser-diode-pumped solid-state laser is designed and manufactured. Separate optical components are adopted in the designed experimental system and students can put these optical components on every place and their ability to establish and adjust optical path can be enhanced. Moreover, experimental education outline of Laser Principle and Its Application is revised and improved. At last, experimental guide book for the designed and manufactured experimental device is writed. The experimental teaching innovation will improve experimental teaching effect and quality of Laser Principle and Its Application.
\end{abstract}

\section{INTRODUCTION}

Laser technology is one of modern optoelectronic technology, and is very important for modern industry[1-2]. Laser Principle and Its Application is a professional basic course for optoelectronic information science and engineering major[3-5], and is very important for students to understand and know well other relative curriculum, and to accomplish professional comrehensive practice and graduation project.

With change of economic situation and ajustment of economic target, china is faced with transconformation from big industral country to powerful industral country, so it is very needed to train a lot of new type talented persons, who not only possess basic manufacture technique but also can innnovate independently. In order to train students having above-mentioned ability, we find that practical education content of Laser Principle and Its Application should be improved. Many education workers have do some work for improving teaching quality about Laser Principle and Its Application ${ }^{[6-8]}$, but there are still many problem needing to be solved.

** Corresponding Author, E-mail:niuchunhui@bistu.edu.cn

** Niu Chun-hui, shanxi province, china, Beijing Information \& Technology University

14th Conference on Education and Training in Optics and Photonics: ETOP 2017, edited by Xu Liu,

Xi-Cheng Zhang, Proc. of SPIE Vol. 10452, 1045250 - @ 2017 ICO, IEEE, OSA, SPIE

CCC code: $0277-786 X / 17 / \$ 18 \cdot$ doi: $10.1117 / 12.2269589$

Proc. of SPIE Vol. 10452 1045250-1 
In past few years, we have worked a lot for improving exerimental education content of Laser Principle and Its Application, some teaching devices are purchased and new experimental guide books are writed. However, the purchased experimental teaching devices on the market have only simle and easy functions, which are all directed towards classroom teaching content. The purchased teaching devices can help students to know basic principle content, but their framwork isn't flexible and it is impossible to train students' comprehensive abilities, for example, to design experimental system, to adjust light path, and to deal with emergency situation.

Aiming at above-mentioned problem, we propose improving experimental education content from experimental teaching devices and experimental guide book. We have designed and manufactured a experimental system, named laser-diode-pumped solid-state laser experiment system. It is different with experimental teaching devices on the market that separate optical components are adopted in the designed experimental system and are fixed on three-dimensional adjusting racks mounted on magnetic base, so students can put these optical components on every place and their ability to establish and adjust optical path can be enhanced. Moreover, we have revised and improved experimental education outline of Laser Principle and Its Application, some experimental teaching content for students to design independently is added. At last, we have writed experimental guide book for the designed and manufactured experimental device.

The paper can be divided into four sections. The first section presents the designed and manufactured experimantal system of laser-diode-pumped solid-state laser. The second section describes experimental teaching content developed from the experimental system. The work of writing experimental guide book is introduced in the third section. A conclusion is given in the last section.

\section{EXPERIMENTAL SYSTEM OF LASER-DIODE-PUMPED}

\section{SOLID-STATE LASER}

Typical structure of laser-diode-pumped solid-state laser is displayed in Fig. 1. It can be seen that the structure can be composed of pumping laser diode (LD), laser crystal, frequency doubling crystal, Q-modulating crystal and resonant cavity (including output mirror and back mirror), where, laser diode emits laser with wavelength of $808 \mathrm{~nm}$ and is taken as pumping light source, and laser crystal can generate $1064 \mathrm{~nm}$ laser. Passing through frequency doubling crystal, 1064nm laser can be tranformed to $532 \mathrm{~nm}$ laser. A Q-modulating crystal can be used to substitute the frequency doubling crystal and to generate $1064 \mathrm{~nm}$ pulse laser with higher peak power and narrower pulse width.

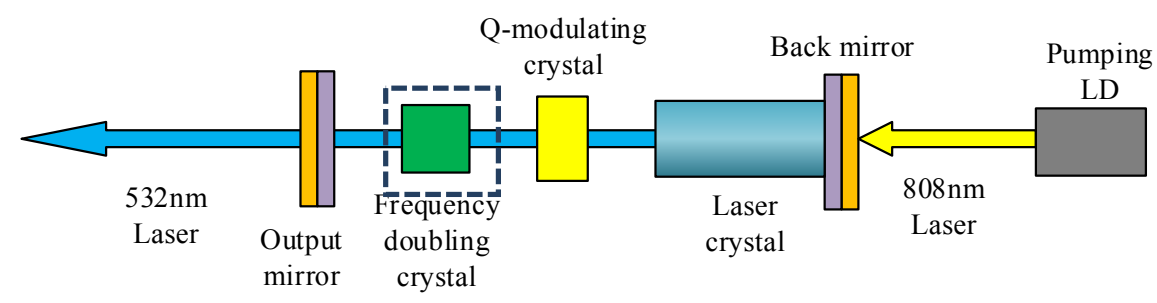

Fig. 1 Schematic diagram of laser-diode-pumped solid-state laser

According to typical laser-diode-pumped solid-state laser and requirement of practical teaching of Laser Principle and Its Application, we designed and manufactured experimental system of laser-diode-pumped solid-state laser, as shown in Fig. 2. In order to make students to know well structure, working principle and operating process of solide-state laser, separate optical components are adopted and fixed on adjusting racks designed by ourself. These adjusting racks can be mounted on 
magnetic bases and move to every place on optical table. One optical component can be taken as one module, and each module can be placed everywhere. These module can be selected to constitute the whole to achieve specially designated function.

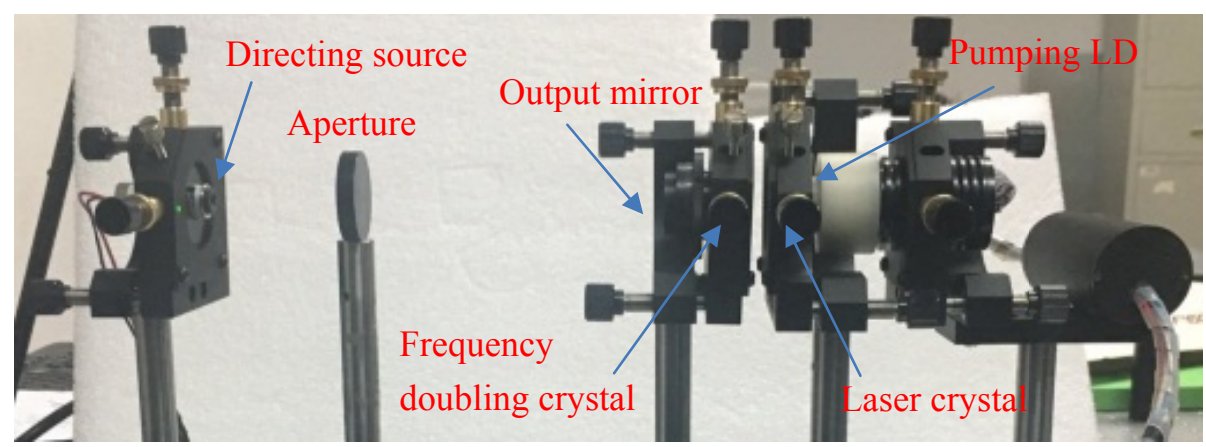

Fig. 2 Experimental system of laser-diode-pumped solid-state laser

It can be seen from Fig.2 that the designed experimental system includes such modules as directing source, aperture, output mirror, frequency doubling crystal, laser crystal and pumping laser diode (LD), where, output mirror is fixed on a magnetic sucking disc and can be attracted on anyone adjusting rack. Directing source emits red laser with wavelength of $650 \mathrm{~nm}$, which is used as directing line for building optical path. The back surface of laser crystal is used as laser back mirror and can reflect $1064 \mathrm{~nm}$ and $532 \mathrm{~nm}$ laser totally, but there is high transmissivity for $808 \mathrm{~nm}$ laser.

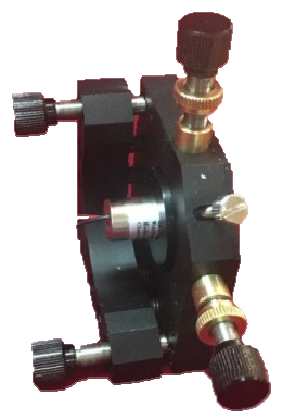

Fig. 3 a designed adjusting rack

Fig . 3 displays the ajusting rack designed for the experimental system elaborately, and it is composed of a locking knob and four adjusting knobs. The locking knob is used to lock optical components. The four ajusting knobs include up-down adjusting knob, left-right adjusting knob, pitching knob and deflecting knob and are used to precisely adjust up-down position, left-right position, pitching angle and deflection angle, respectively. For improving adjusting precision but, at the same time, preventing screw dislocation, screw pitch of four adjusting knobs are set as $0.1 \mathrm{~mm}$. Moreover, a special design is adopted to increase adjusting scope of optical components in longitudinal direction to $10 \mathrm{~cm}$.

\section{EXPERIMENTAL TEACHING CONTENT}

According to our teaching experience, many experimental teaching content can be set by using above-mentioned experimental system. Here, main experimental teaching content is described as follows. 


\subsection{Experiment of measuring I-P curve of laser diode}

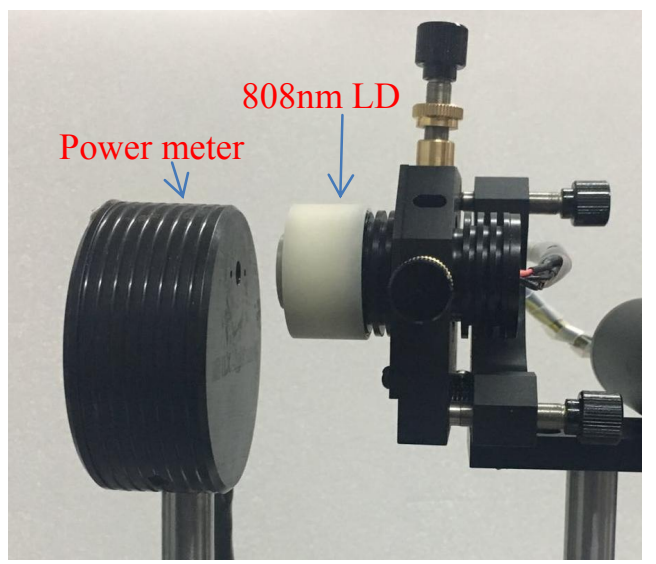

Fig. 4 Experimental installation for measuring I-P curve of laser diode

According to optical path shown in Fig.4, 808nm LD is fixed and power meter is placed at laser outlet in front of LD. Working current of $808 \mathrm{~nm}$ LD is adjusted from zero to maximal value, and reading of power meter and driving current of $808 \mathrm{~nm}$ LD are recorded successively. I-P curve can be drawn according to these recorded data and is used to obtain threshold current of 808nm LD.

\subsection{Experiment of designing and adjusting resonant cavity of solid-state laser}
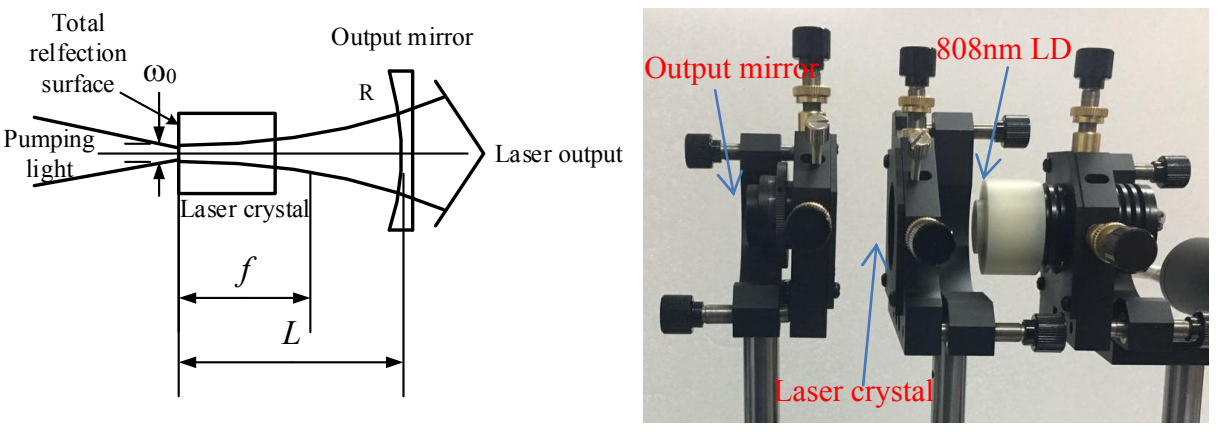

Fig. 5 Structure of resonant cavity of solid-state laser

Fig. 5 show structure of resonant cavity of solid-state laser, here, the left is principle diagram and the right is practical experimental installation. The back surface of laser crystal is a total reflection surface for $1064 \mathrm{~nm}$ and $532 \mathrm{~nm}$ laser, but is transparent for $808 \mathrm{~nm}$ laser.

As shown in the left diagram of Fig. 5, structure of resonant cavity is a flat-concave cavity, whose structure parameters are:

$$
g_{1}=1-\frac{L}{R_{1}}=1, \quad g_{2}=1-\frac{L}{R_{2}}
$$

here, $L$ is length of resonant cavity, $R_{2}$ and $R_{1}$ are radius of output mirror and back mirror, respectively.

According to stability situation of laser resonant cavity, when $0<g_{1} g_{2}<1$, a resonant cavity is a stable cavity, so when $\mathrm{L}<R_{2}$, the resonant cavity in Fig.5 is stable.

Moreover, laser waist locates at front surface of laser crystal in the left diagram in Fig. 5. The laser waist can be described as 


$$
\omega_{0}=\sqrt{\frac{\left[L\left(R_{2}-L\right)\right]^{\frac{1}{2}} \lambda}{\pi}} .
$$

In our designed experimental system, $R_{2}=250 \mathrm{~mm}$ and $\mathrm{L}=100 \mathrm{~mm}$, so the laser waist $\omega_{0}$ can be caculated. Light spot of pumping $808 \mathrm{~nm}$ LD on the input surface of laser crystal should be less than $\omega_{0}$, so that pattern matching between pumping laser and oscillating basic-mode laser can be realized and laser at basic mode can be generated easily.

At first, $808 \mathrm{~nm}$ pumping LD is located at the right side of light path of resonant cavity, and directing laser with wavelength of $650 \mathrm{~nm}$ is fixed on the left side of light path, adjusting up-down knob, left-right knob, pitching knob and deflecting knob make $650 \mathrm{~nm}$ laser point at center of $808 \mathrm{~nm}$ LD. Then, laser output mirror is placed at front of laser crystal and the surface coated reflection film is orientated towards laser crystal. Adjusting knob on adjusting rack of output mirror make the reflected 650nm laser go back into light outlet of $650 \mathrm{~nm}$ LD. Subsequently, working current of $808 \mathrm{~nm}$ pumping LD is increased above $600 \mathrm{~mA}$, and a infrared display card is placed in the front of output mirror to detect whether or not there is $1064 \mathrm{~nm}$ laser. If no, Adjusting knob of output mirror is adjusted endlessly until $1064 \mathrm{~nm}$ laser is generated.

Through above-mentioned adjusting process, students can master skill of adjusting optical path. Furthermore, students can also observe output power of $1064 \mathrm{~nm}$ laser by adjusting length of resonant cavity, so they will have concrete cognition about stability situation of resonant cavity.

\subsection{Experiment of measuring output power and energy productivity of 1064nm laser}

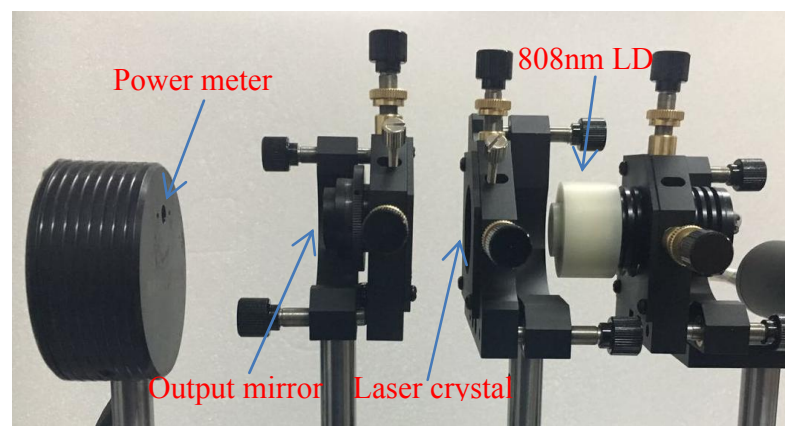

Fig. 6 Set-up diagram for measuring output power

Fig. 6 displays set-up diagram for measuring output power. After keeping length of laser resonant cavity, output mirror is adjusting until $1064 \mathrm{~nm}$ laser is generated, then power meter is used to detect output power. Adjusting position and angle of output mirror, laser crystal, $808 \mathrm{~nm} \mathrm{LD}$ in turn make output power of $1064 \mathrm{~nm}$ laser largest, then the power value is recorded. According to recorded data, relationship curve between output power of $1064 \mathrm{~nm}$ laser and pumping power of $808 \mathrm{~nm}$ laser can be fitted, so that P-P conversion efficiency and threshold pumping power can be obtained. 


\subsection{Experiment of study frequency doubling effect of solid-state laser}

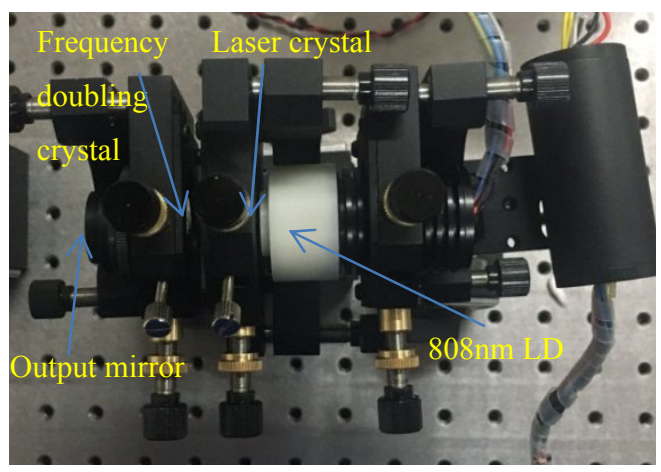

Fig. 7 Set-up diagram for observing frequency doubling effect of solid-state laser

According to light path of intracavity frequency doubling shown in Fig. 7, a frequency doubling crystal is inserted into resonant cavity of $1064 \mathrm{~nm}$ laser, then adjusting knobs of adjusting racks of each optical component make green output laser with 532nm wavelength strongest. Through this experiment, students can know operating method and working principle of frequency doubling crystal. Moreover, the frequency doubling crystal can be rotated along optical axis so as to change phase angle of crystal, which will make students know well about phase matching condition of frequency doubling crystal.

\subsection{Experiment of observing output mode of 532nm laser}

After obtaining highest output power of 532nm laser according to adjusting method described in section 2.4. Transverse mode of $532 \mathrm{~nm}$ laser can be observed through a white screen, which will change with adjustment of output mirror. Severel recorded transverse modes are displayed in Fig. 8. Through the experiment, students can observe output transverse modes of 532nm laser intuitively and know relationship between laser mode with resonant cavity parameters.

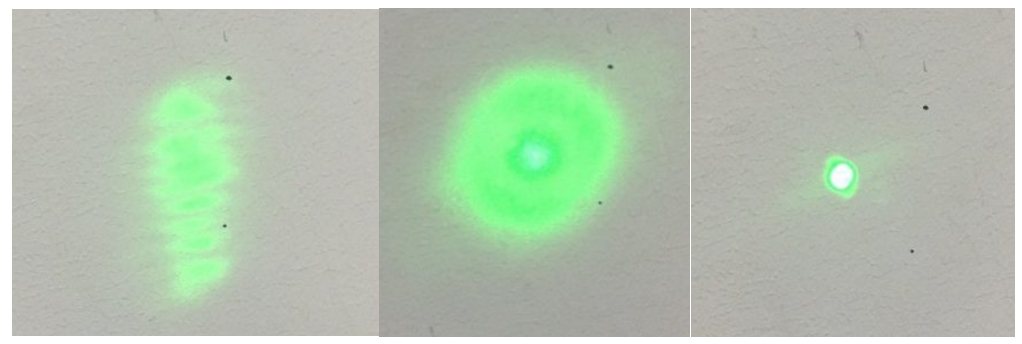

Fig. 8 Several output transverse modes of 532nm laser

\subsection{Experiment of Q-modulation of solid-state laser}

There are mainly three Q-modulating methods such as electro-optical Q-modulation, acousto-optic Q-modulation and passive saturable absorber Q-modulation. A sort of saturable absorber, named $\mathrm{Cr}^{4+}: \mathrm{YAG}, \quad$ is adopted in our designed experimental system. It has advantage of simple structure, being convenient to use, no electromagnetic interference and being able to obtain giant pulse with high peak power and small pulse width.

As shown in Fig. 9, freqency doubling crystal is substituted by passive Q-modulating crystal. Working current of 808nm pumping LD is adjusted about 1A, then position and angle of Q-modulating crystal is adjusted until a light spot of 1064nm laser appears on infrared display card in front of laser output mirror. 


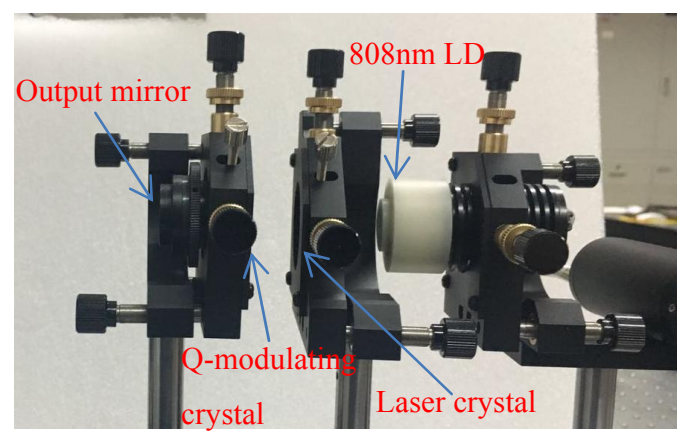

Fig. 9 Set-up diagram for Q-modulation output of 1064nm laser

Q-modulation output power of $1064 \mathrm{~nm}$ laser will be measured by using power meter and be recored. Through changing length of resonant cavity, relationship between Q-modulation output power and cavity length weill be obtained. Students can know Q-modulation principle and its influence factor.

\section{REVISION OF EXPERIMENTAL GUIDE BOOK}

Aiming at faced problem in experimental teaching process and according to designed and manufactured experimental system of laser-diode-pumped solid-state laser, the experimental guide book is revised. The concrete revised content includes: 1) to revise and improve experimental teaching outline of Laser Principle and Its Application and to add above-mentioned experimental content into outline according to requirement;2) to increase experimental teaching time from six hours to eight hours; 3 ) to carefully select experiment items, these experiment content capable of displaying in classroom teaching, for example, constitution of laser resonant cavity, coupled modes of LD-pumping system and output modes of LD, should be cut off; 4) to reduce demonstration type experiments and to increase synthesizing type experiments; 5) to arrange all experiments according to degree of difficulty and to specify compulsory experiments and optional experiments; 6) to design thinking questions for students to ask before, in or after experiment.

\section{CONCLUSION}

In this paper, experimental education content of Laser Principle and Its Application is proposed to improve from two aspects : experimental teaching devices and experimental guide book. At first, a experimental system of laser-diode-pumped solid-state laser is designed and manufactured, and separate optical components are adopted in the designed experimental system, so students can put these optical components on every place, which make their ability to establish and adjust optical path be enhanced. Secondly, experimental education outline of Laser Principle and Its Application is revised and improved for requirement. In the end, experimental guide book for the designed and manufactured experimental device is writed. We hope that the experimental teaching innovation will improve experimental teaching effect and quality of Laser Principle and Its Application. 


\section{ACKNOWLEDGMENT}

This paper is supported by 2016 year curriculum construction project of Beijing information science \& technology university under grant numbers 2016KGYB13 and 2016KGYB14 .

\section{REFERENCES}

[1] Gao Y., " Analysis of state-of-the-art and application prospect of laser”, China Science and Technology Information, (19), 270-271 (2007).

[2] Zheng Q., Zhao L. and Qian L. S., "Application and development of laser-diode-pumping solid-state laser with high power", Optical Precision Engineering, 9 (1), 6-9 (2001).

[3] Anthony E. S., [Laser], Oxford University Press, (1986).

[4] William T. S., [Laser fundamentals] (second edition), Cambridge University Press, (2004) .

[5] Orazio S., [Principles of Lasers] (Fourth Edition), Plenum Press (New York and London), (1998).

[6] Ju G. F., "Exploration and practice about experimental teaching innnovation of Laser Principle", Journal of Guangdong University of Technology (Social Science Edition), 5(9), 140-141 (2005).

[7] Gao ZH. H.,Qiu W. Q., Li J. ZH. and An Y. Y., "Study about multimedia teaching system of Laser principle", Optical Technology (Supplement), 28 (8),223-224 (2002).

[8] Hu SH. M. and Luo H., "Teaching experience about Laser Principle", Journal of Higher Education Research, 35 (3), 71-73 (2012). 